\title{
A new efficient technique for solving modified Chua's circuit model with a new fractional operator
}

\author{
Manuel De la Sen', Sinan Deniz ${ }^{2^{*}}$ (D) and Hasan Sözen ${ }^{3}$
}

\author{
"Correspondence: \\ sinandeniz01@gmail.com \\ ${ }^{2}$ Department of Mathematics, \\ Faculty of Art and Sciences, Manisa \\ Celal Bayar University, 45140 \\ Manisa, Turkey \\ Full list of author information is \\ available at the end of the article
}

\begin{abstract}
Chua's circuit is an electronic circuit that exhibits nonlinear dynamics. In this paper, a new model for Chua's circuit is obtained by transforming the classical model of Chua's circuit into novel forms of various fractional derivatives. The new obtained system is then named fractional Chua's circuit model. The modified system is then analyzed by the optimal perturbation iteration method. Illustrations are given to show the applicability of the algorithms, and effective graphics are sketched for comparison purposes of the newly introduced fractional operators.
\end{abstract}

Keywords: Optimal perturbation iteration method; Fractional Chua's circuit model; Caputo; Caputo-Fabrizio; Atangana-Baleanu

\section{Introduction}

Over the last two decades, the interest in fractional derivatives and their implementations has been intensified. Various physical phenomena in technological developments have been determined in terms of fractional derivatives or differential equations. Moreover, the modeling of many engineering problems relies on fractional operators. There are many reasons for the need for fractional calculus, but the most important ones can be stated as the singular kernel with locality and also the nonsingular kernel with nonlocality. In order to overcome these problems, Baleanu and Atangana came up with new fractional operators, namely Atangana-Baleanu $(\mathrm{AB})$ derivative operators, with fractional order based upon the recognized Mittag-Leffler function [1]. Those noninteger derivatives have all the usefulness of those of past provisions. These trend ideas on operators have got the attention of many mathematicians and scientists. AB derivatives have been used for analyzing engine oil based generalized Brinkman-type nano-liquid with molybdenum disulfide nanoparticles of spherical shape [2]. Modeling and analysis of the fractional HBV model with the Atangana-Baleanu derivative have been studied in [3]. These operators have been also applied to nanofluids to enhance the performance of solar collectors, and the same researchers have also compared Atangana-Baleanu and Caputo-Fabrizio fractional models [4]. The logarithmic-KdV equation involving Mittag-Leffler type kernel with $\mathrm{AB}$ derivative has been investigated in [5]. AB derivatives have been used for numer-

(c) The Author(s) 2021. This article is licensed under a Creative Commons Attribution 4.0 International License, which permits use, sharing, adaptation, distribution and reproduction in any medium or format, as long as you give appropriate credit to the original author(s) and the source, provide a link to the Creative Commons licence, and indicate if changes were made. The images or other third party material in this article are included in the article's Creative Commons licence, unless indicated otherwise in a credit line to the material. If material is not included in the article's Creative Commons licence and your intended use is not permitted by statutory regulation or exceeds the permitted use, you will need to obtain permission directly from the copyright holder. To view a copy of this licence, visit http://creativecommons.org/licenses/by/4.0/. 
ical patterns in the reaction-diffusion system [6]. Adams-Bashforth scheme has been formulated with Atangana-Baleanu-Caputo fractional derivative to model chaotic problems [7]. Three different fractional derivatives including $A B C$ have been considered to solve the Korteweg-de Vries and Korteweg-de Vries-Burger equations [8]. ABC-fractional masks have been designed in image processing [9]. In addition to $\mathrm{ABC}$ derivatives, CaputoFabrizio fractional operators have been also used by many authors. Transient response of the parallel RCL circuit by using the Caputo-Fabrizio fractional derivative has been analyzed by Rezapour et al. in [10]. Other works can also be counted as in [11-15]. In addition to all these articles, readers are advised to read the works in [16-35].

Most of the dynamical systems have long-range temporal memory. Modeling of these systems with fractional-order derivatives has more advantages than classical models such as in [36-38]. The main reason for using fractional derivatives in these models is the memory concept. Generally, when the output of a system at each time $t$ depends only on the input at time $t$, such systems are called memoryless systems. On the other hand, when the system has to remember previous values of the input in order to determine the current value of the output, such systems are said to be non-memoryless systems or memory systems. One of the well-known memoryless systems is the Markov chain phenomena. Human decision making or shape memory alloy are non-memoryless systems [39]. For instance, in [36], a biological model a fractional-order model for Ebola virus infection with delayed immune response on heterogeneous complex networks has been investigated. It has been demonstrated that Chua's model with fractional derivative with a lower order 2.7 has been able to replicate a chaotic attractor [37]. The model of the Wien bridge oscillator has been also analyzed within the scope of fractional calculus, and the results obtained therein showed that a limit cycle can be replicated for any fractional order, with an adequate value of the amplifier gain [38]. The jerk model has been investigated using the concept of fractional derivative, and a chaotic attractor has been obtained with the system order as low as 2.1 [40].

One of the basic mathematical systems is Chua's circuit system as shown in Fig. 1. This typical nonlinear electronic circuit and modified models have been investigated by many authors. Hartley et al. analyzed chaos in fractional-order Chua's system [37]. Controlling spiral waves in a model of two-dimensional arrays of Chua's circuits have been investigated in [41]. Some analytical and numerical methods for hidden attractors' localization have been proposed in [42]. This system has been solved also for comparison purposes of the Atangana-Baleanu and Caputo-Fabrizio derivatives [40]. Analyzing the circuit using Kirchhoff's circuit laws, the dynamics of Chua's circuit can be accurately modeled by means of a system of three nonlinear ordinary differential equations in the variables $x(t)$,

Figure 1 Classical Chua's system

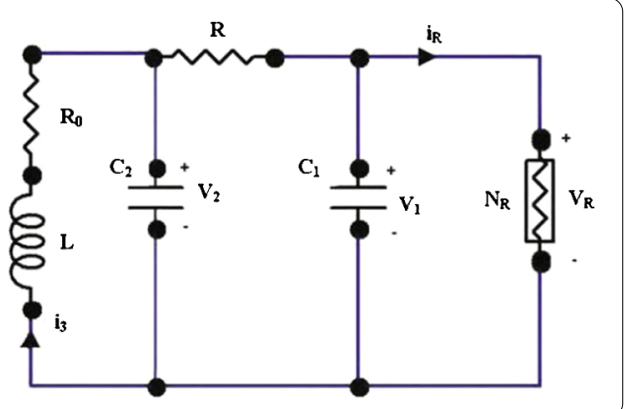


$y(t)$, and $z(t)$, which represent the voltages across the capacitors $C_{1}$ and $C_{2}$ and the electric current in the inductor $L$ respectively. As in the papers [37, 40,41], a mathematical model of Chua's circuit model can be displayed as follows:

$$
\begin{aligned}
& \frac{x(\tau)}{d \tau}=\theta[y(\tau)-x(\tau)-f(x(\tau))], \\
& R C_{2} \frac{y(\tau)}{d \tau}=x(\tau)-y(\tau)+R z(\tau), \\
& \frac{z(\tau)}{d \tau}=-\phi y(\tau),
\end{aligned}
$$

where $f(x(\tau))=m x(\tau)+\frac{1}{2}(n-m)(|x(\tau)+1|-|x(\tau)-1|)$ demonstrates the electrical response of the nonlinear resistor. $\theta$ and $\phi$ are parameters determined by the particular values of the circuit components. The shape of $f(x(\tau))$ relies on the rigorous model on its components. In this paper we try to solve the above system by using the optimal perturbation iteration algorithms (OPIAs) and different fractional operators. This technique has been applied to many types of ODEs and PDEs such as Bratu-type [43], Riccati differential equation [44], heat transfer equations [45], nonlinear systems [46], Lane-Emden types [47], generalized regularized long wave equations [48]. More studies can also be found in [49-57]. The rest of the current paper is planned as follows. In Sect. 2, we give some basic concepts of the optimal perturbation iteration algorithms, and we apply this technique to systems of ordinary differential equations. In Sect. 3, we propose some modifications to the classical Chua circuit by using trend fractional derivatives. In Sect. 4, two illustrations of some wellknown problems are carried out to validate the proposed method. Finally, a conclusion part is given in the last section.

\section{Formulation of OPIAs for systems of ordinary differential equations}

Deniz and Bildik presented the optimal perturbation iteration algorithms (OPIA) in 2016 [43]. It has been proven that these algorithms can be safely applied to many types of differential equations [44-52]. In this section, we give a short description of OPIA for systems of ordinary differential equation. Then we will be able to generate OPIA for the modified fractional Chua's circuit model (MFCCM) in the next section. The modified fractional Chua's circuit model can be converted into a standard model by replacing the fractional numbers with integer ones.

Let us consider the following system of ODEs:

$$
A_{k}\left(\dot{x}_{k}, x_{j}, \epsilon, \tau\right)=0 ; \quad k=1,2, \ldots, K ; j=1,2, \ldots, K .
$$

Here, $k$ represents the number of ODEs in Eq. (2.1). Clearly, this system can be rewritten as

$$
\begin{aligned}
& A_{1}=A_{1}\left(\dot{x}_{1}, x_{1}, x_{2}, x_{3}, \ldots, x_{K}, \epsilon, \tau\right)=0, \\
& A_{2}=A_{2}\left(\dot{x}_{2}, x_{1}, x_{2}, x_{3}, \ldots, x_{K}, \epsilon, \tau\right)=0, \\
& \vdots \\
& A_{k}=A_{k}\left(\dot{x}_{k}, x_{1}, x_{2}, x_{3}, \ldots, x_{K}, \epsilon, \tau\right)=0 .
\end{aligned}
$$


By taking first correction term in the classical perturbation series, an approximate solution of the system will be considered as

$$
x_{k, n+1}=x_{k, n}+\varepsilon x_{k, n}^{c}
$$

where $n$ denotes the $n$ th-order approximate solution to be obtained and $x_{k, n}^{c} \mathrm{~s}$ are the correction terms. By using Taylor series expansions in the neighborhood of $\varepsilon=0$, we can approximate this system as follows:

$$
A_{k}=\sum_{m=0}^{M} \frac{1}{m !}\left[\left(\frac{d}{d \varepsilon}\right)^{m} A_{k}\right]_{\varepsilon=0} \varepsilon^{m} ; \quad k=1,2, \ldots, K,
$$

where

$$
\frac{d}{d \varepsilon}=\frac{\partial \dot{x}_{k, n+1}}{\partial \varepsilon} \frac{\partial}{\partial \dot{x}_{k, n+1}}+\sum_{j=1}^{K}\left(\frac{\partial \dot{x}_{k, n+1}}{\partial \varepsilon} \frac{\partial}{\partial \dot{x}_{k, n+1}}\right)+\frac{\partial}{\partial \varepsilon}
$$

is given for the $(n+1)$ th iteration

$$
A_{k}\left(\dot{x}_{k, n+1}, x_{j, n+1}, \epsilon, \tau\right)=0 \text {. }
$$

Furnishing Eq. (2.5) into (2.4), an iteration algorithm will be established as follows:

$$
A_{k}=\sum_{m=0}^{M} \frac{1}{m !}\left[\left(\dot{x}_{k, n}^{c} \frac{\partial}{\partial \dot{x}_{k, n+1}}+\sum_{j=1}^{K}\left(\dot{x}_{j, n}^{c} \frac{\partial}{\partial \dot{x}_{j, n+1}}\right)+\frac{\partial}{\partial \varepsilon}\right)^{m} H_{k}\right]_{\varepsilon=0} \varepsilon^{m}=0 .
$$

Equation (2.7) is a first-order ordinary differential equation. Therefore, the solution can be found for the correction terms $x_{k, n}^{c}$. After that, by using Eq. $(2.3)$, the $(n+1)$ th approximation can be reached. Iterations can be stopped when an adequate approximation is achieved.

In this work, we give a novel technique for PIAs to ameliorate the approximations. First of all, we insert convergence-control parameters $p_{i},(i=0,1, \ldots)$ into Eq. (2.3) to get the following functions:

$$
\begin{aligned}
& x_{k, 1}(t ; p)=x_{k, 0}+p_{0} x_{k, 0}^{c}, \\
& x_{k, 2}(t ; p)=x_{k, 1}+p_{1} x_{k, 1}^{c}, \\
& \vdots \\
& x_{k, m+1}(t ; p)=x_{k, n}+p_{m} x_{k, m}^{c} .
\end{aligned}
$$

It is obvious that Eq. (2.8) will reduce to ordinary PIM when all of the parameters $p_{m}$ are one. In order to find an optimal value of $p$, we substitute the approximate solution $x_{m}$ into 
Eq. (2.1). Thus, the following residual will be obtained:

$$
\begin{aligned}
& \operatorname{Res}_{A_{1}}=A_{1}\left(\dot{x}_{1, m}, x_{1, m}, x_{2, m}, \ldots, x_{K, m}, p_{0}, \epsilon, t\right)=0, \\
& \operatorname{Res}_{A_{2}}=A_{2}\left(\dot{x}_{2}, x_{1, m}, x_{2, m}, \ldots, x_{K, m}, p_{1}, \epsilon, t\right)=0, \\
& \vdots \\
& \operatorname{Res}_{A_{k}}=A_{k}\left(\dot{x}_{k}, x_{1, m}, x_{2, m}, \ldots, x_{K, m}, p_{m}, \epsilon, t\right)=0 .
\end{aligned}
$$

One can easily say that when $\operatorname{Res}\left(t ; p_{m}\right)=0, x_{m}(t ; p)$ is the wanted solution of the given problem. Otherwise, we can compute the following functional:

$$
\begin{aligned}
& \operatorname{JRes}_{A_{1}}\left(p_{1}\right)=\int_{\Theta}\left(\operatorname{Res}_{A_{1}}\right)^{2}\left(t ; p_{0}\right) d t, \\
& \operatorname{JRes}_{A_{2}}\left(p_{2}\right)=\int_{\Theta}\left(\operatorname{Res}_{A_{2}}\right)^{2}\left(t ; p_{1}\right) d t, \\
& \vdots \\
& \operatorname{JRes}_{A_{k}}\left(p_{K}\right)=\int_{\Theta}\left(\operatorname{Res}_{A_{k}}\right)^{2}\left(t ; p_{K-1}\right) d t,
\end{aligned}
$$

where $\Theta$ is the physical domain of the considered equations. By doing so, the optimal values for parameters $p$ can be reached by solving

$$
\frac{d J \operatorname{Res}_{A_{1}}}{d p_{1}}=0, \quad \frac{d J \operatorname{Res}_{A_{2}}}{d p_{2}}=0, \quad \ldots, \quad \frac{d J \operatorname{Res}_{A_{K}}}{d p_{K}}=0
$$

\section{OPIA for MCCM with different fractional derivatives}

In this part, we give a brief formulation of OPIA for modified Chua's circuit model (MCCM). For this reason, we use Caputo (C), Caputo-Fabrizio (CF), and AtanganaBaleanu $(\mathrm{ABC})$ fractional operators, respectively.

\subsection{Algorithm with Caputo fractional operator}

The standard systems can be turned into the fractional differential forms. Equivalent systems for classical form of Chua's circuit model by Caputo derivatives can be reached by changing the derivatives $D_{\tau}^{\beta} x, D_{\tau}^{\beta} y, D_{\tau}^{\beta} z$ by ${ }_{0}^{C} D_{\tau}^{\beta} x,{ }_{0}^{C} D_{\tau}^{\beta} y$, and ${ }_{0}^{C} D_{\tau}^{\beta} z$, respectively. Note here that $0<\beta \leq 1$. With these changes, we have the following new forms:

$$
\begin{aligned}
& { }_{0}^{C} D_{\tau}^{\beta} x(\tau)-\theta[y(\tau)-x(\tau)-f(x(\tau))]=0, \\
& R C_{2}{ }_{0}^{C} D_{\tau}^{\beta} y(\tau)-x(t)+y(t)-R z(t)=0, \\
& { }_{0}^{C} D_{\tau}^{\beta} z(\tau)+\phi y(\tau)=0,
\end{aligned}
$$

where

$$
{ }_{0}^{C} D_{\tau}^{\beta} x=\frac{1}{\Gamma(1-\beta)} \int_{0}^{\tau}(\tau-t)^{-\beta} x^{\prime}(t) d t
$$




$$
\begin{aligned}
& { }_{0}^{C} D_{\tau}^{\beta} y=\frac{1}{\Gamma(1-\beta)} \int_{0}^{\tau}(\tau-t)^{-\beta} y^{\prime}(t) d t, \\
& { }_{0}^{C} D_{\tau}^{\beta} z=\frac{1}{\Gamma(1-\beta)} \int_{0}^{\tau}(\tau-t)^{-\beta} z^{\prime}(t) d t .
\end{aligned}
$$

We can represent the above equations in a closed form as follows:

$$
\begin{aligned}
& F_{1}\left({ }_{0}^{C} D_{\tau}^{\beta} x, x, y, z, \varepsilon\right)=0, \\
& F_{2}\left({ }_{0}^{C} D_{\tau}^{\beta} y, x, y, z, \varepsilon\right)=0, \\
& F_{3}\left({ }_{0}^{C} D_{\tau}^{\beta} z, y, \varepsilon\right)=0,
\end{aligned}
$$

where $\varepsilon=1$ is the artificial perturbation parameter which can be inserted into Eqs. (3.1)(3.3) as follows:

$$
\begin{aligned}
& { }_{0}^{C} D_{\tau}^{\beta} x(\tau)-\theta[y(\tau)-x(\tau)-\varepsilon f(x(\tau))]=0, \\
& R C_{2}^{C} D_{\tau}^{\beta} y(\tau)-x(t)+y(t)-\varepsilon R z(t)=0, \\
& { }_{0}^{C} D_{\tau}^{\beta} z(\tau)+\phi \varepsilon y(\tau)=0 .
\end{aligned}
$$

Processing as in Sect. 2, the first approximate solution in the perturbation series can be taken as

$$
\begin{aligned}
& x_{n+1}=x_{n}+\varepsilon\left(x_{c}\right)_{n}, \\
& y_{n+1}=y_{n}+\varepsilon\left(y_{c}\right)_{n}, \\
& z_{n+1}=z_{n}+\varepsilon\left(z_{c}\right)_{n}
\end{aligned}
$$

with only one correction term. Here, $n \in \mathrm{N} \cup\{0\}$ and $\left(x_{c}\right)_{n},\left(y_{c}\right)_{n},\left(z_{c}\right)_{n}$ are $n$th correction terms. Then, by replacing Eq. (3.6) into (3.4) and using the Taylor series, we get

$$
\begin{aligned}
& F_{1}+F_{1{ }_{0} D_{\tau}^{\beta} x}\left(\left(x_{c}\right)_{n}\right) \varepsilon+F_{1 x}\left(\left(x_{c}\right)_{n}\right) \varepsilon+F_{1 y}\left(y_{c}\right)_{n} \varepsilon+F_{1 z}\left(z_{c}\right)_{n} \varepsilon+F_{1 \varepsilon} \varepsilon=0, \\
& F_{2}+F_{2 C_{0} D_{\tau}^{\beta} y}\left(\left(y_{c}\right)_{n}\right) \varepsilon+F_{2 x}\left(\left(x_{c}\right)_{n}\right) \varepsilon+F_{2 y}\left(y_{c}\right)_{n} \varepsilon+F_{2 z}\left(z_{c}\right)_{n} \varepsilon+F_{2 \varepsilon} \varepsilon=0, \\
& F_{3}+F_{3{ }_{0} D_{\tau}^{\beta} z}\left(\left(z_{c}\right)_{n}\right) \varepsilon+F_{3 y}\left(y_{c}\right)_{n} \varepsilon+F_{3_{\varepsilon}} \varepsilon=0,
\end{aligned}
$$

where

$$
F_{1 x}=\frac{\partial F_{1}}{\partial x}, \quad F_{1 y}=\frac{\partial F_{1}}{\partial y}, \quad F_{2 z}=\frac{\partial F_{2}}{\partial z}, \quad F_{1 \varepsilon}=\frac{\partial F_{1}}{\partial \varepsilon},
$$

\subsection{Algorithm with Caputo-Fabrizio fractional operator}

By performing the same changes as in the previous subsection, we obtain the new fractional case for the new modified system via changing $D_{\tau}^{\beta} x, D_{\tau}^{\beta} y, D_{\tau}^{\beta} z$ by ${ }_{0}^{\mathrm{CF}} D_{\tau}^{\beta} x,{ }_{0}^{\mathrm{CF}} D_{\tau}^{\beta} y$, and ${ }_{0}^{\mathrm{CF}} D_{\tau}^{\beta} z$, respectively. Therefore, new Caputo-Fabrizio $(\mathrm{C}-\mathrm{F})$ fractional forms will be

$$
\begin{aligned}
& { }_{0}^{\mathrm{CF}} D_{\tau}^{\beta} x(\tau)-\theta[y(\tau)-x(\tau)-f(x(\tau))]=0, \\
& R C_{20}^{\mathrm{CF}} D_{\tau}^{\beta} y(\tau)-x(t)+y(t)-R z(t)=0, \\
& { }_{0}^{\mathrm{CF}} D_{\tau}^{\beta} z(\tau)+\phi y(\tau)=0 .
\end{aligned}
$$


${ }_{0}^{\mathrm{CF}} D_{\rho}^{\beta}$ represents $C-F$ fractional derivatives of order $\beta$, and it is given by

$$
\begin{aligned}
& { }_{0}^{\mathrm{CF}} D_{\tau}^{\beta} x(t)=\frac{M(\beta)}{1-\beta} \int_{0}^{\tau} \exp \left(\frac{-\beta(\tau-t)}{1-\beta}\right) D x(t) d t, \\
& { }_{0}^{\mathrm{CF}} D_{\tau}^{\beta} y(\tau)=\frac{M(\beta)}{1-\beta} \int_{0}^{\tau} \exp \left(\frac{-\beta(\tau-t)}{1-\beta}\right) D y(t) d t, \\
& { }_{0}^{\mathrm{CF}} D_{\tau}^{\beta} z(\tau)=\frac{M(\beta)}{1-\beta} \int_{0}^{\tau} \exp \left(\frac{-\beta(\tau-t)}{1-\beta}\right) D z(t) d t,
\end{aligned}
$$

where $M(\beta)$ is a normalization function such that $M(0)=M(1)=1$.

Applying similar procedures, one can get the following:

$$
\begin{aligned}
& F_{1}+F_{1 \mathrm{CF}_{D}^{\beta} D_{\tau}^{\beta} x}\left(\left(x_{c}\right)_{n}\right) \varepsilon+F_{1 x}\left(\left(x_{c}\right)_{n}\right) \varepsilon+F_{1 y}\left(y_{c}\right)_{n} \varepsilon+F_{1 z}\left(z_{c}\right)_{n} \varepsilon+F_{1 \varepsilon} \varepsilon=0, \\
& F_{2}+F_{2{ }_{0} D_{\tau}^{\beta} y}\left(\left(y_{c}\right)_{n}\right) \varepsilon+F_{2 x}\left(\left(x_{c}\right)_{n}\right) \varepsilon+F_{2 y}\left(y_{c}\right)_{n} \varepsilon+F_{2 z}\left(z_{c}\right)_{n} \varepsilon+F_{2 \varepsilon} \varepsilon=0, \\
& F_{3}+F_{3{ }_{0} D_{\tau}^{\beta} z}^{\beta}\left(\left(z_{c}\right)_{n}\right) \varepsilon+F_{3 y}\left(y_{c}\right)_{n} \varepsilon+F_{3 \varepsilon} \varepsilon=0 .
\end{aligned}
$$

\subsection{Algorithm with Atangana-Baleanu fractional operator}

As a final work, we consider the ABC derivatives [1] form of the MCCM with the similar changes $D_{\tau}^{\beta} x, D_{\tau}^{\beta} y, D_{\tau}^{\beta} z$ by ${ }_{0}^{\mathrm{ABC}} D_{\tau}^{\beta} x,{ }_{0}^{\mathrm{ABC}} D_{\tau}^{\beta} y$, and ${ }_{0}^{\mathrm{ABC}} D_{\tau}^{\beta} z$. Thus, the new modified system can be obtained as follows:

$$
\begin{aligned}
& { }_{0}^{\mathrm{ABC}} D_{\tau}^{\beta} x(\tau)-\theta[y(\tau)-x(\tau)-f(x(\tau))]=0, \\
& R C_{20}^{\mathrm{ABC}} D_{\tau}^{\beta} y(\tau)-x(t)+y(t)-R z(t)=0, \\
& { }_{0}^{\mathrm{ABC}} D_{\tau}^{\beta} z(\tau)+\phi y(\tau)=0 .
\end{aligned}
$$

Here, ${ }_{0}^{\mathrm{ABC}} D_{\tau}^{\beta}(\cdot)$ stands for an $\mathrm{ABC}$ fractional derivative in the Liouville-Caputo sense of order $\beta$ and can be given as

$$
\begin{aligned}
& { }_{0}^{\mathrm{ABC}} D_{\tau}^{\beta} x(t)=\frac{M(\beta)}{1-\beta} \int_{0}^{\tau} E_{\beta}\left(\frac{-\beta(\tau-t)}{1-\beta}\right) D x(\tau) d t, \\
& { }_{0}^{\mathrm{ABC}} D_{\tau}^{\beta} y(t)=\frac{M(\beta)}{1-\beta} \int_{0}^{\tau} E_{\beta}\left(\frac{-\beta(\tau-t)}{1-\beta}\right) D y(\tau) d t, \\
& { }_{0}^{\mathrm{ABC}} D_{\tau}^{\beta} z(t)=\frac{M(\beta)}{1-\beta} \int_{0}^{\tau} E_{\beta}\left(\frac{-\beta(\tau-t)}{1-\beta}\right) D z(\tau) d t,
\end{aligned}
$$

where

$$
E_{\beta}(z)=\sum_{k=0}^{\infty} \frac{z^{k}}{\Gamma(\beta k+1)}
$$


is the famous Mittag-Leffler function. Likewise, using the Taylor series with perturbation theorem, one can get

$$
\begin{aligned}
& F_{1}+F_{0}^{F_{1 \mathrm{ABC}} D_{\tau}^{\beta} x}\left(\left(x_{c}\right)_{n}\right) \varepsilon+F_{1 x}\left(\left(x_{c}\right)_{n}\right) \varepsilon+F_{1 y}\left(y_{c}\right)_{n} \varepsilon+F_{1 z}\left(z_{c}\right)_{n} \varepsilon+F_{1 \varepsilon} \varepsilon=0, \\
& F_{2}+F_{0}^{F_{0}^{\mathrm{ABC}} D_{\tau}^{\beta} y}\left(\left(y_{c}\right)_{n}\right) \varepsilon+F_{2 x}\left(\left(x_{c}\right)_{n}\right) \varepsilon+F_{2 y}\left(y_{c}\right)_{n} \varepsilon+F_{2 z}\left(z_{c}\right)_{n} \varepsilon+F_{2 \varepsilon} \varepsilon=0, \\
& F_{3}+F_{3_{0}^{\mathrm{ABC}} D_{\tau}^{\beta} z}^{\beta}\left(\left(z_{c}\right)_{n}\right) \varepsilon+F_{3 y}\left(y_{c}\right)_{n} \varepsilon+F_{3 \varepsilon} \varepsilon=0 .
\end{aligned}
$$

We will call all of Eqs. (3.7), (3.11), and (3.16) OPIAs for the corresponding systems with interested derivatives. By making the necessary computations at $\varepsilon=0$ for Eqs. (3.1)-(3.3), we can reach any order of OPIAs. Guess functions $u_{0}, v_{0}$ should be chosen fittingly according to the given conditions to begin the iteration procedures. Accordingly, first correction functions $\left(u_{c}\right)_{0},\left(v_{c}\right)_{0}$ are evaluated using the OPIAs with $x_{0}, y_{0}, z_{0}$ and the initial conditions. After that, the first approximations $u_{1}, v_{1}$ can be established by knowing $x_{0}, y_{0}, z_{0}$ with $\left(x_{c}\right)_{0},\left(y_{c}\right)_{0},\left(z_{c}\right)_{0}$ and so on. To advance the correctness of the solutions and capability of the proposed method, the concept of optimal parameters may be used. Subsequently, using the optimal parameters, we can evaluate the errors with the aid of residuals (2.10).

\section{Illustration}

In this section, we deal with MCCM (3.1)-(3.3) based on the aforementioned fractional operators.

Example 4.1 Consider the following modified nonlinear Chua's circuit system [37]:

$$
\begin{aligned}
& { }_{0}^{\Lambda} D_{\tau}^{\beta} x(\tau)=\theta\left[y+\frac{x-2 x^{3}}{7}\right], \\
& { }_{0}^{\Lambda} D_{\tau}^{\beta} y(\tau)=x-y+z, \\
& { }_{0}^{\Lambda} D_{\tau}^{\beta} z(\tau)=\phi y,
\end{aligned}
$$

where $\Lambda$ is the corresponding fractional operator.

Closed and perturbed form of the above system can be written as follows:

$$
\begin{aligned}
& { }_{0}^{\Lambda} D_{\tau}^{\beta} x(\tau)-\theta\left[y+\varepsilon \frac{x-2 x^{3}}{7}\right]=0, \\
& { }_{0}^{\Lambda} D_{\tau}^{\beta} y(\tau)-x+y-z=0, \\
& { }_{0}^{\Lambda} D_{\tau}^{\beta} z(\tau)-\phi y=0 .
\end{aligned}
$$

By taking the one correction term from the classical perturbation expansion, the approximate solution can be taken as follows:

$$
\begin{aligned}
& x_{n+1}=x_{n}+\varepsilon\left(x_{c}\right)_{n}, \\
& y_{n+1}=y_{n}+\varepsilon\left(y_{c}\right)_{n}, \\
& z_{n+1}=z_{n}+\varepsilon\left(z_{c}\right)_{n} .
\end{aligned}
$$




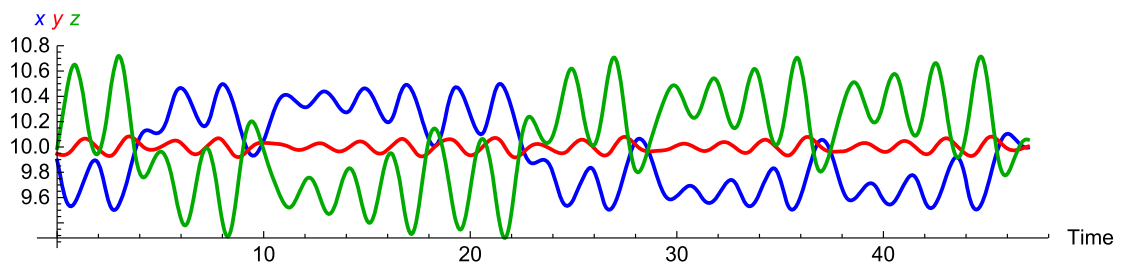

(a) Simulations with Caputo derivative.

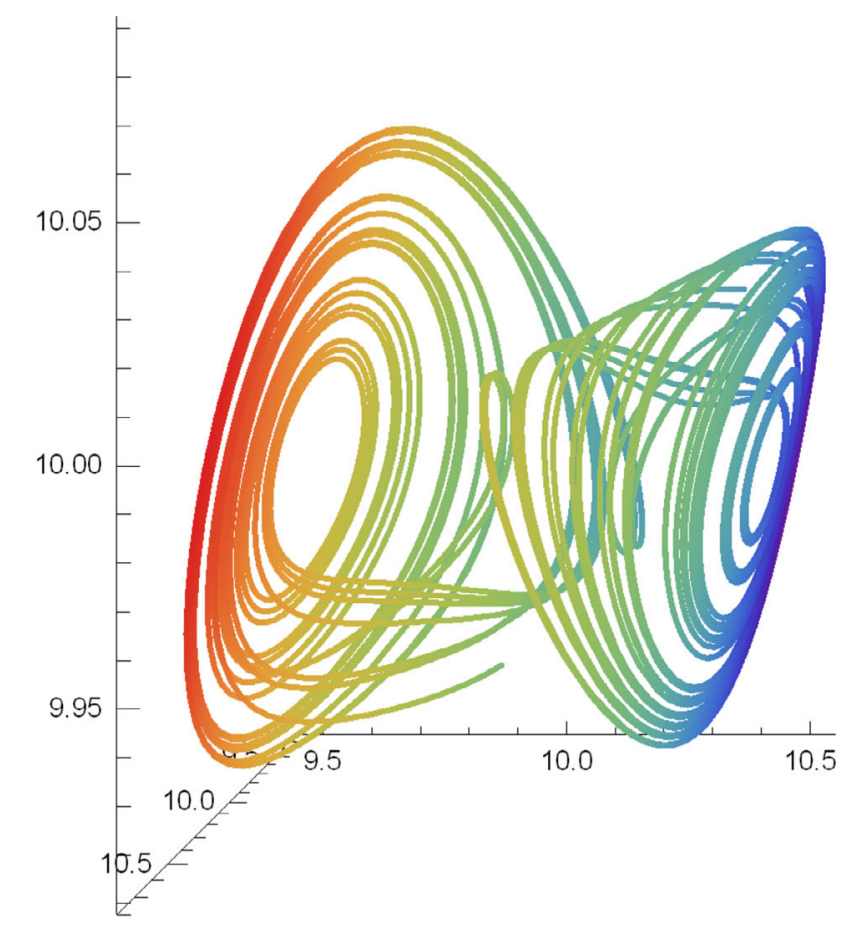

(b) Chaotic attractor.

Figure 2 OPIM - C solutions of the modified Chua's circuit in Example 4.1

By inserting (4.3) into the closed form of the system and by using algorithms (3.7), (3.11), and (3.16), one can construct the related functional equations for the system with the aid of residual (2.9), optimal parameters $p_{1}, p_{2}, p_{3}$ can be computed as mentioned in Sect. 2.

One can see the results of third-order OPIA solutions from Figs. 2-4. Figure 2 displays the semi-analytical solutions and attractors for $x(0)=9.95, y(0)=9.88, z(0)=9.79, \beta=$ 0.95, $\theta=14, \phi=10$ for derivatives in the Caputo sense.

By using algorithm (3.11) with $x(0)=9.81, y(0)=9.84, z(0)=9.99, \beta=0.95, \theta=11, \phi=$ 20, OPIA solutions in the Caputo-Fabrizio (C-F) sense can be sketched as in Fig. 3.

From Fig. 4, one can see the semi-analytical solutions and attractors for $x(0)=9.93$, $y(0)=10.012, z(0)=9.94, \beta=0.95, \theta=13, \phi=21$ for derivatives in the $\mathrm{ABC}$ sense. 


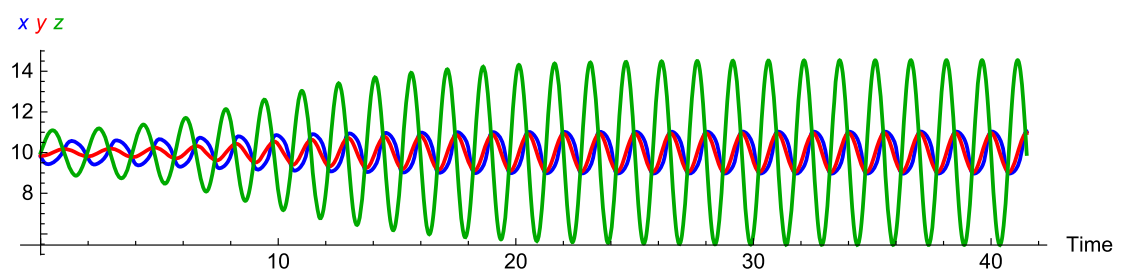

(a) Simulations with C-F derivative.

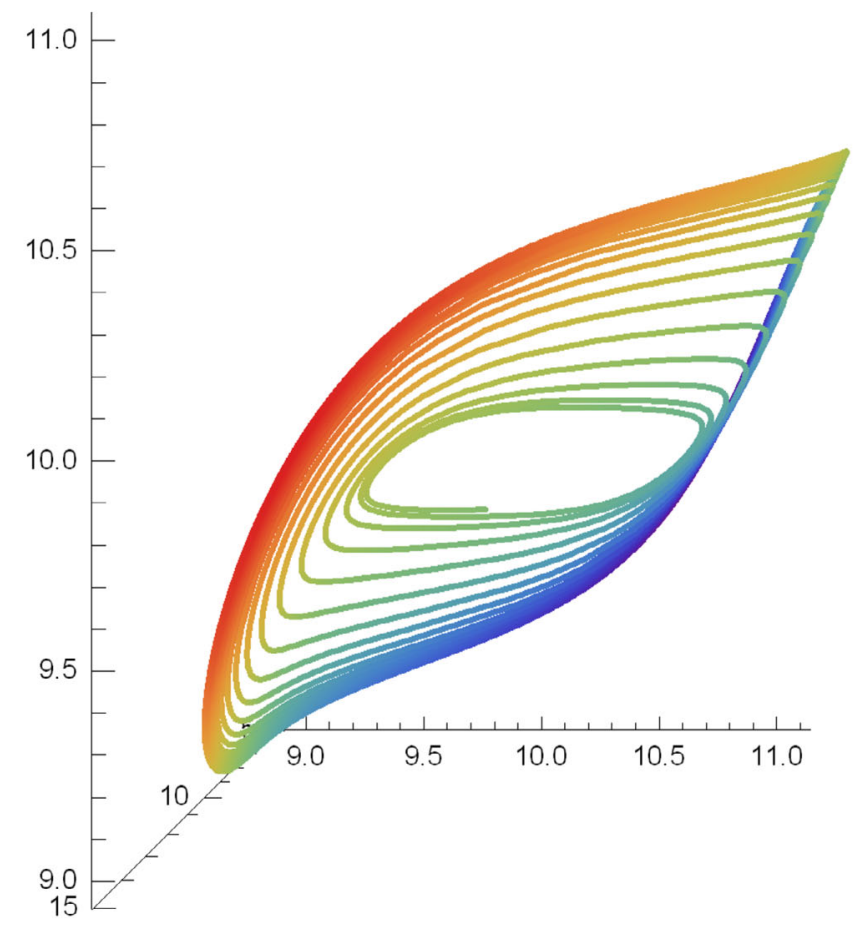

(b) Chaotic attractor.

Figure 3 OPIM - CF solutions of the modified Chua's circuit in Example 4.1

Example 4.2 As a second example, we consider the modified Chua's circuit in [40]. In order to compare our results, we will benefit from the solution of numerical techniques such as the Adams-Bashforth rule as in [7].

To start the iterations, we can use the same initial conditions as $x(0)=0.6, y(0)=0.3$, $z(0)=-0.5$ as in [40].

Absolute residual errors, or briefly ARE, of $x(\tau)$ for all of three fractional forms are given in Table 1 at $\beta=0.9$ and for some specific values of $\tau$. Tables 2 and 3 show the AREs obtained by using the fractional operators for $y(\tau)$ and $z(\tau)$, respectively.

From Figs. 5-7, we can detect all of the AREs obtained by sixth-order approximation and various operators plotted by $(\bullet),(\boldsymbol{\Lambda})$, and (-) for Caputo, Caputo-Fabrizio and Atangana-Baleanu derivatives at $\beta=0.7$. Figures $8-10$ show the same draws for $\beta=0.8$. 


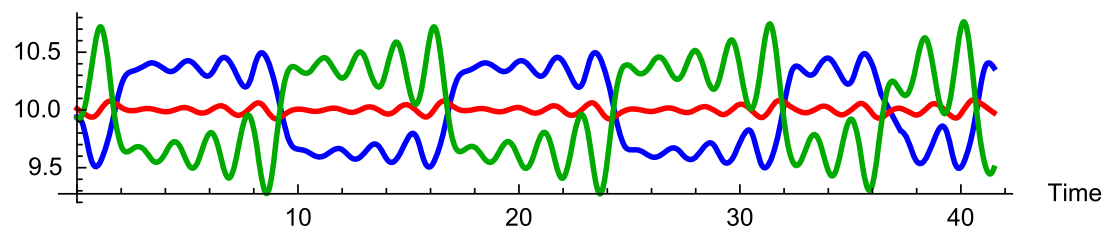

(a) Simulations with $\mathrm{ABC}$ derivative.

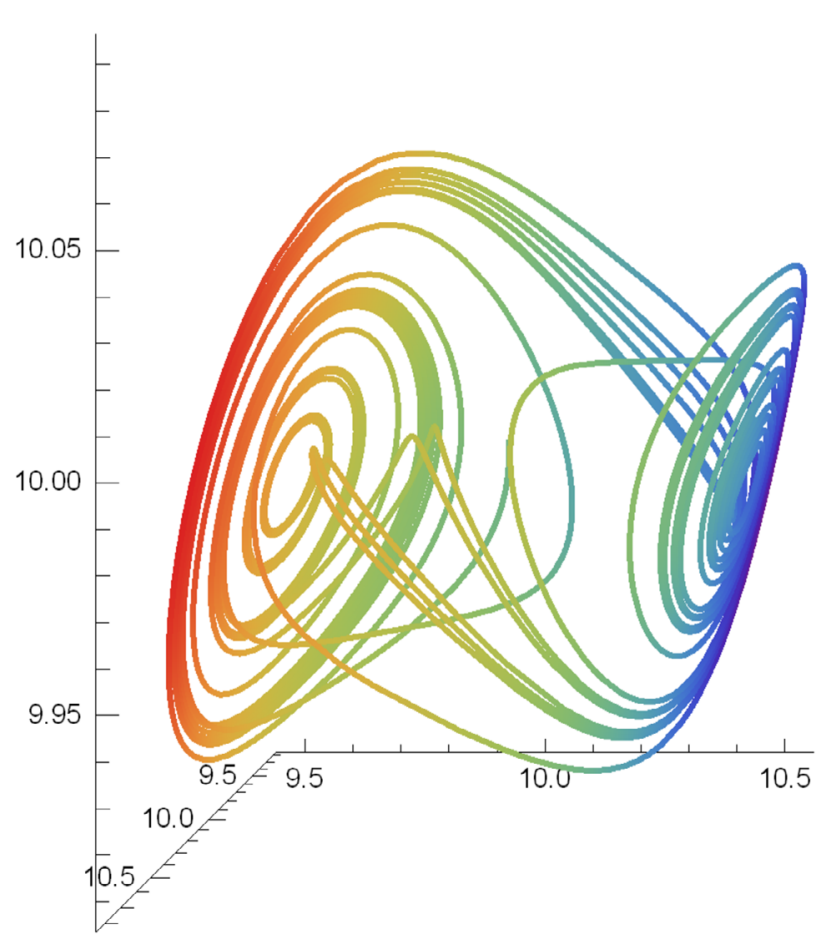

(b) Chaotic attractor.

Figure 4 OPIM - ABC solutions of the modified Chua's circuit in Example 4.1

Table 1 AREs of $x(\tau)$ obtained by fourth-order OPIA solutions using different fractional operators at $\beta=0.9$

\begin{tabular}{llll}
\hline$\tau$ & Error for C & Error for CF & Error for ABC \\
\hline 0.1 & 0.000218839 & 0.000164539 & 0.000142742 \\
0.2 & 0.000478919 & 0.000360979 & 0.000313472 \\
0.3 & 0.000786097 & 0.000593972 & 0.000516311 \\
0.4 & 0.00114697 & 0.000868771 & 0.000755924 \\
0.5 & 0.00156897 & 0.00119131 & 0.00103758 \\
0.6 & 0.00206044 & 0.00156828 & 0.00136724 \\
0.7 & 0.0026308 & 0.00200722 & 0.00175161 \\
0.8 & 0.0032906 & 0.00251663 & 0.00219827 \\
0.9 & 0.0040517 & 0.00310609 & 0.00271577 \\
\hline
\end{tabular}

\section{Conclusion}

In this research paper, we have applied the optimal perturbation iteration method to analyze the modified fractional Chua's circuit model (MFCCM) semi-analytically. We change 
Table 2 AREs of $y(\tau)$ obtained by fourth-order OPIA solutions using different fractional operators at $\beta=0.9$

\begin{tabular}{llll}
\hline$\tau$ & Error for C & Error for CF & Error for ABC \\
\hline 0.1 & 0.000186291 & 0.000131828 & 0.000120903 \\
0.2 & 0.000408297 & 0.000289647 & 0.000265774 \\
0.3 & 0.000671168 & 0.000477306 & 0.000438184 \\
0.4 & 0.000980718 & 0.000699162 & 0.000642171 \\
0.5 & 0.00134351 & 0.000960141 & 0.000882311 \\
0.6 & 0.00176692 & 0.00126581 & 0.00116377 \\
0.7 & 0.00225927 & 0.00162246 & 0.0014924 \\
0.8 & 0.00282994 & 0.00203717 & 0.00187478 \\
0.9 & 0.00348945 & 0.00251795 & 0.00231836 \\
\hline
\end{tabular}

Table 3 AREs of $x(\tau)$ obtained by fourth-order OPIA solutions using different fractional operators at $\beta=0.9$

\begin{tabular}{llll}
\hline$\tau$ & Error for C & Error for CF & Error for ABC \\
\hline 0.1 & 0.000088061 & 0.0000770918 & 0.0000661117 \\
0.2 & 0.000193869 & 0.000169805 & 0.000145692 \\
0.3 & 0.000320109 & 0.000280515 & 0.000240801 \\
0.4 & 0.000469827 & 0.000411917 & 0.000353776 \\
0.5 & 0.000646473 & 0.000567071 & 0.000487271 \\
0.6 & 0.000853958 & 0.000749441 & 0.000644296 \\
0.7 & 0.00109671 & 0.000962954 & 0.000828261 \\
0.8 & 0.00137972 & 0.00121205 & 0.00104303 \\
0.9 & 0.00170866 & 0.00150175 & 0.00129296 \\
\hline
\end{tabular}

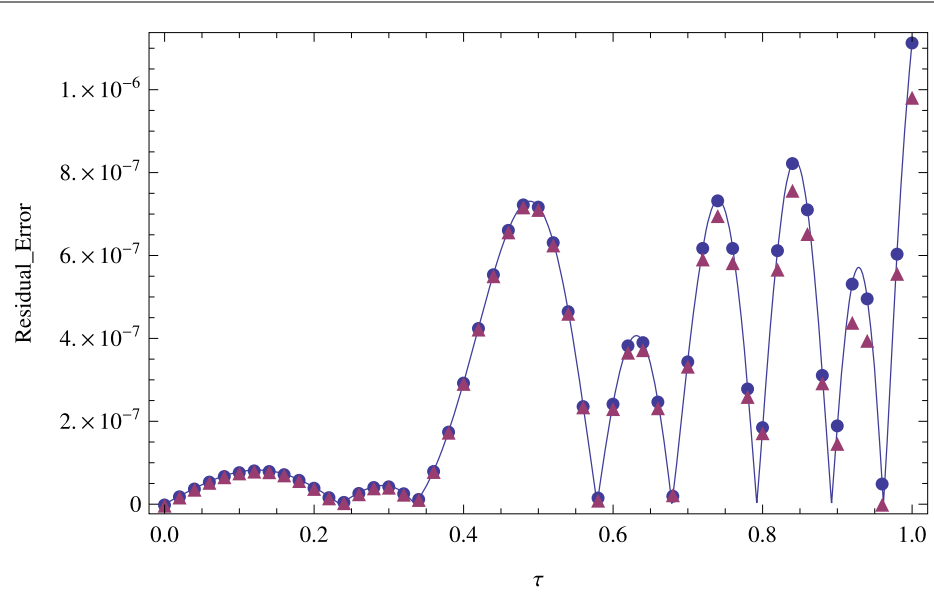

Figure 5 ARES by sixth-order approximations at $\beta=0.7, C(\bullet), C F(\boldsymbol{\Lambda})$, and ABC $(-)$ for $x(\tau)$

the classical forms of MFCCM with fractional ones with the help of three different fractional operators. Two specific examples of modified fractional Chua's circuit model are solved by the presented technique. Figure 2 shows the OPIM solutions and attractors for $x(0)=9.95, y(0)=9.88, z(0)=9.79$ and different values of parameters. Figures 3 and 4 display the semi-analytical solutions when the same algorithm is applied with $x(0)=9.81$, $y(0)=9.84, z(0)=9.99$ in the $(\mathrm{C}-\mathrm{F})$ sense and $x(0)=9.93, y(0)=10.012, z(0)=9.94$ in the ABC sense, respectively. Also, absolute residual errors are sketched in Figs. 5-7 by using three operators and various values of parameters. One can say that there is no big difference in using different operators for solving this system. The results are nearly the same for 


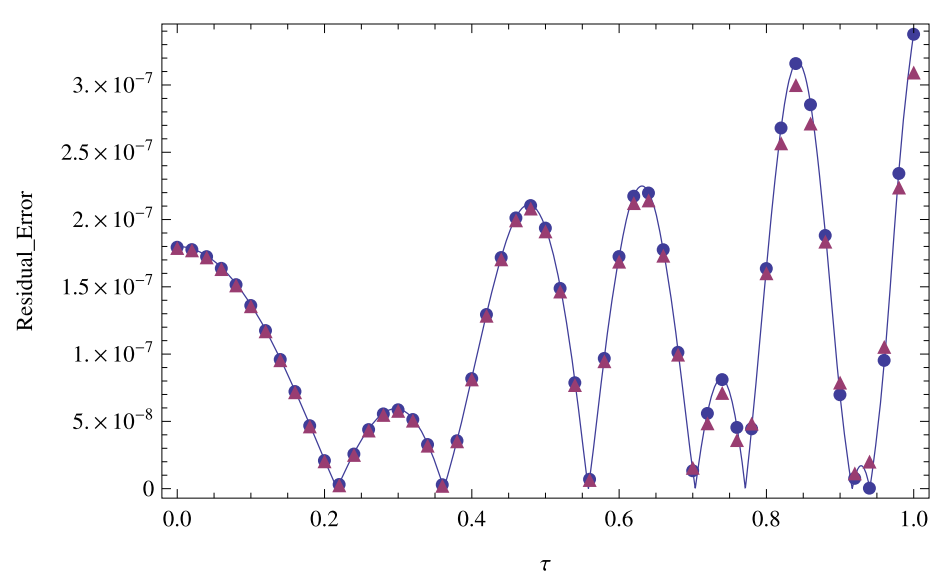

Figure 6 ARES by sixth-order approximations at $\beta=0.7, C(\bullet), C F(\mathbf{\Lambda})$, and ABC (-) for $y(\tau)$

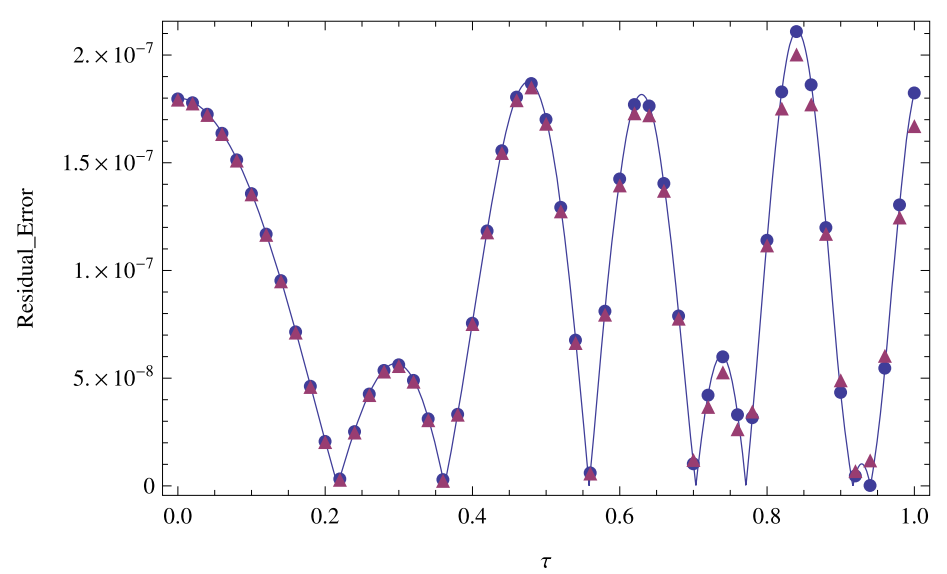

Figure 7 ARES by sixth-order approximations at $\beta=0.7, C(\bullet), C F(\boldsymbol{\Lambda})$, and ABC $(-)$ for $z(\tau)$

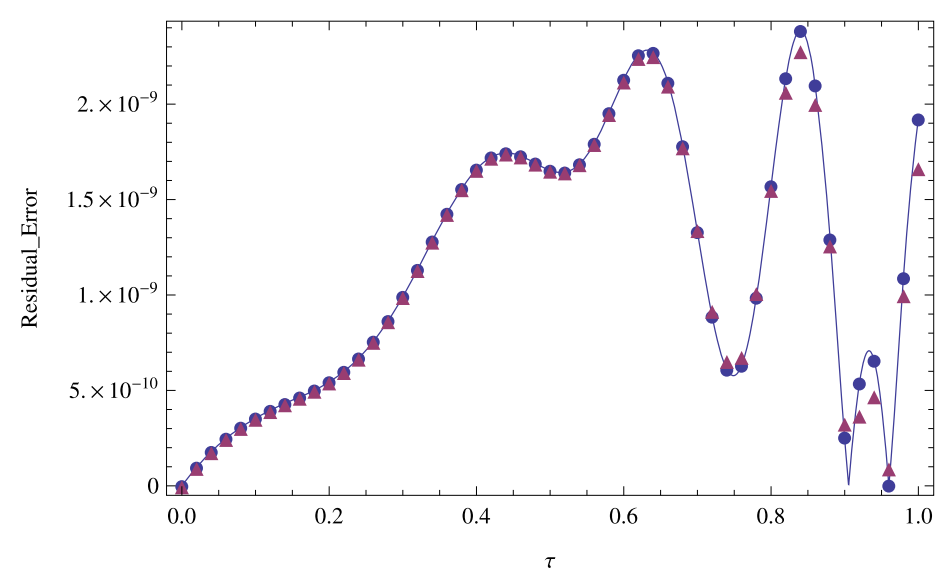

Figure 8 ARES by tenth-order approximations at $\beta=0.8, C(\bullet), C F(\boldsymbol{\Lambda})$, and ABC (-) for $x(\tau)$ 


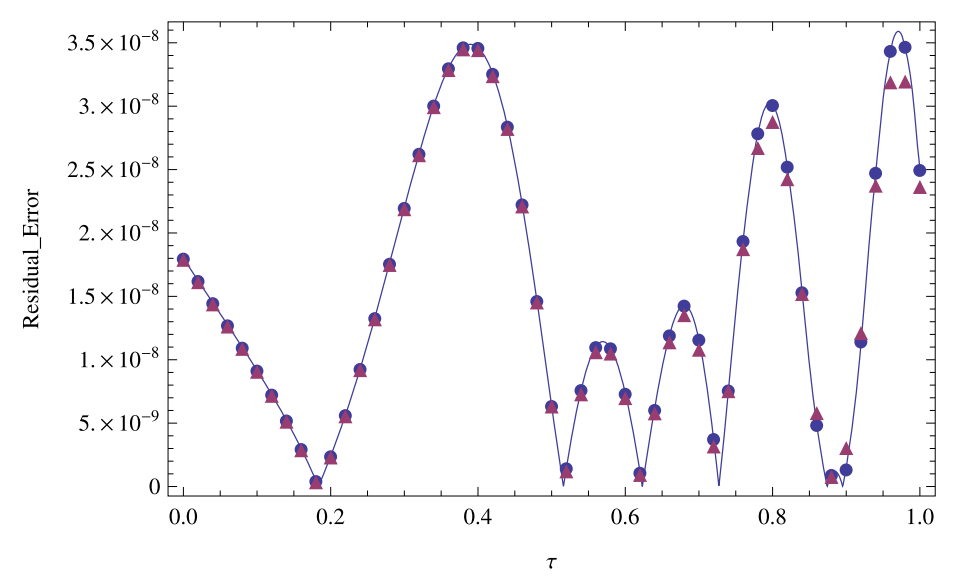

Figure 9 ARES by tenth-order approximations at $\beta=0.8, C(\bullet), C F(\boldsymbol{\Lambda})$, and ABC (-) for $y(\tau)$

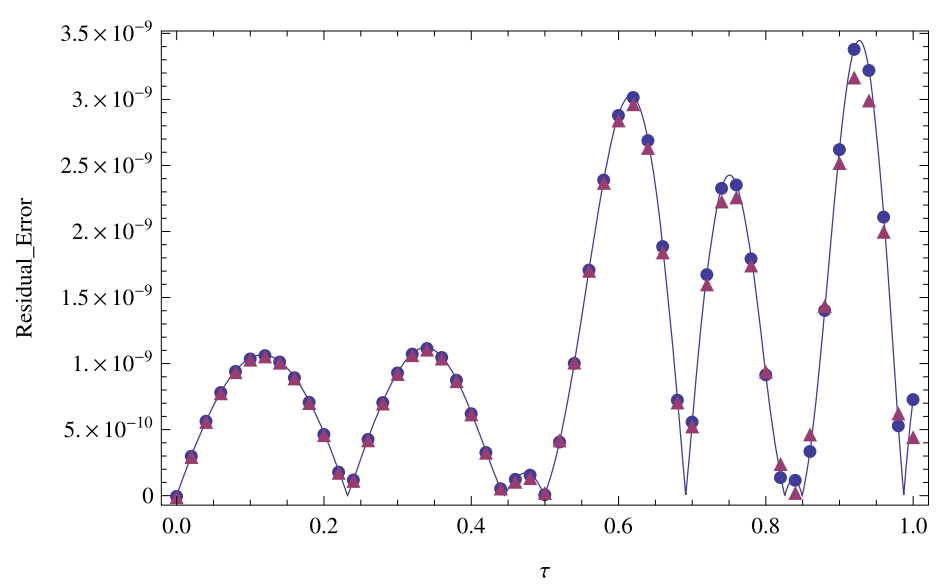

Figure 10 ARES by tenth-order approximations at $\beta=0.8, C(\bullet), C F(\mathbf{\Lambda})$, and ABC $(-)$ for $z(\tau)$

all three derivatives, and they are indistinguishable on graphics. In addition, illustrations show the availability and usefulness of the OPIAs for the system of differential equations.

\section{Acknowledgements}

The authors would like to thank the anonymous reviewers very much for their positive comments, careful reading, and useful suggestions on improving this article.

Funding

The authors are grateful to the Spanish Government for Grant RTI2018-094336-B-I00 (MCIU/AEI/FEDER, UE) and to the Basque Government for Grant IT1207-19.

Availability of data and materials

Please contact the corresponding author for data requests.

\section{Competing interests}

The authors declare that they have no competing interests.

Authors' contributions

The authors contributed equally to this paper. All authors read and approved the final manuscript. 


\section{Author details}

1 Department of Electricity and Electronics, Faculty of Science and Technology, Institute of Research and Development of Processes_IIDP, University of the Basque Country_UPV/EHU, Bo Sarriena s/n (48080), 48940 Leioa, Bizkaia, Spain. ${ }^{2}$ Department of Mathematics, Faculty of Art and Sciences, Manisa Celal Bayar University, 45140 Manisa, Turkey. ${ }^{3}$ Department of Electrical and Electronics Engineering, Faculty of Engineering, Manisa Celal Bayar University, 45140 Manisa, Turkey.

\section{Publisher's Note}

Springer Nature remains neutral with regard to jurisdictional claims in published maps and institutional affiliations.

Received: 28 September 2020 Accepted: 10 December 2020 Published online: 07 January 2021

\section{References}

1. Atangana, A., Baleanu, D.: New fractional derivatives with nonlocal and non-singular kernel: theory and application to heat transfer model. arXiv preprint arXiv:1602.03408 (2016)

2. Jan, S.A.A., et al.: Engine oil based generalized Brinkman-type nano-liquid with molybdenum disulphide nanoparticles of spherical shape: Atangana-Baleanu fractional model. Numer. Methods Partial Differ. Equ. 34(5), 1472-1488 (2018)

3. Ullah, S., Khan, M.A., Farooq, M.: Modeling and analysis of the fractional HBV model with Atangana-Baleanu derivative. Eur. Phys. J. Plus 133(8), 313 (2018)

4. Sheikh, N.A., et al.: On the applications of nanofluids to enhance the performance of solar collectors: a comparative analysis of Atangana-Baleanu and Caputo-Fabrizio fractional models. Eur. Phys. J. Plus 132(12), 540 (2017)

5. Inc, M., et al.: Investigation of the logarithmic-KdV equation involving Mittag-Leffler type kernel with Atangana-Baleanu derivative. Phys. A, Stat. Mech. Appl. 506, 520-531 (2018)

6. Owolabi, K.M.: Numerical patterns in reaction-diffusion system with the Caputo and Atangana-Baleanu fractional derivatives. Chaos Solitons Fractals 115, 160-169 (2018)

7. Owolabi, K.M., Atangana, A.: On the formulation of Adams-Bashforth scheme with Atangana-Baleanu-Caputo fractional derivative to model chaotic problems. Chaos, Interdiscip. J. Nonlinear Sci. 29(2), 023111 (2019)

8. Saad, K.M., Baleanu, D., Atangana, A.: New fractional derivatives applied to the Korteweg-de Vries and Korteweg-de Vries-Burger's equations. Comput. Appl. Math. 37(4), 5203-5216 (2018)

9. Ghanbari, B., Atangana, A.: A new application of fractional Atangana-Baleanu derivatives: designing ABC-fractional masks in image processing. Phys. A, Stat. Mech. Appl. 542, 123516 (2020)

10. Alizadeh, S., Baleanu, D., Rezapour, S.: Analyzing transient response of the parallel RCL circuit by using the Caputo-Fabrizio fractional derivative. Adv. Differ. Equ. 2020(1), 55 (2020)

11. Aydogan, M.S., Baleanu, D., Mousalou, A., Rezapour, S.: On high order fractional integro-differential equations including the, Caputo-Fabrizio derivative. Bound. Value Probl. 2018(1), 90 (2018)

12. Baleanu, D., Mousalou, A., Rezapour, S.: A new method for investigating approximate solutions of some fractional integro-differential equations involving the Caputo-Fabrizio derivative. Adv. Differ. Equ. 2017(1), 51 (2017)

13. Baleanu, D., Mousalou, A., Rezapour, S.: On the existence of solutions for some infinite coefficient-symmetric, Caputo-Fabrizio fractional integro-differential equations. Bound. Value Probl. 2017(1), 145 (2017)

14. Baleanu, D., Rezapour, S., Mohammadi, H.: Some existence results on nonlinear fractional differential equations. Philos. Trans. R. Soc., Math. Phys. Eng. Sci. 371(1990), 20120144 (2013)

15. Baleanu, D., Rezapour, S., Saberpour, Z:: On fractional integro-differential inclusions via the extended fractional, Caputo-Fabrizio derivation. Bound. Value Probl. 2019(1), 79 (2019)

16. Bildik, N., Deniz, S., Saad, K.M.: A comparative study on solving fractional cubic isothermal auto-catalytic chemical system via new efficient technique. Chaos Solitons Fractals 132, 109555 (2020)

17. Deniz, S.: Semi-analytical analysis of Allen-Cahn model with a new fractional derivative. Math. Methods Appl. Sci. To appear. https://doi.org/10.1002/mma.5892

18. Saad, K.M., Deniz, S., Baleanu, D.: On the new fractional analysis of Nagumo equation. Int. J. Biomath. 12(03), 1950034 (2019)

19. Bildik, N., Deniz, S.: A new fractional analysis on the polluted lakes system. Chaos Solitons Fractals 122, 17-24 (2019)

20. Podlubny, I.: Fractional Differential Equations: An Introduction to Fractional Derivatives, Fractional Differential Equations, to Methods of Their Solution and Some of Their Applications. Mathematics in Science and Engineering, vol. 198. Academic Press, San Diego (1999)

21. Kilbas, A.A., Srivastava, H.M., Trujillo, J.J.: Theory and Applications of Fractional Differential Equations. North-Holland Mathematical Studies, vol. 204. Elsevier, Amsterdam (2006)

22. Li, Z.-B., He, J.-H.: Fractional complex transform for fractional differential equations. Math. Comput. Appl. 15(5), 970-973 (2010)

23. Agarwal, R.P., Lakshmikantham, V., Nieto, J.J.: On the concept of solution for fractional differential equations with uncertainty. Nonlinear Anal., Theory Methods Appl. 72(6), 2859-2862 (2010)

24. Atangana, A.: Non validity of index law in fractional calculus: a fractional differential operator with Markovian and non-Markovian properties. Phys. A, Stat. Mech. Appl. 505, 688-706 (2018)

25. Singh, J., et al.: A fractional epidemiological model for computer viruses pertaining to a new fractional derivative. Appl. Math. Comput. 316, 504-515 (2018)

26. Baskonus, H.M., Bulut, H., Atangana, A.: On the complex and hyperbolic structures of the longitudinal wave equation in a magneto-electro-elastic circular rod. Smart Mater. Struct. 25(3), 035022 (2016)

27. Khan, Altaf, M., Atangana, A.: Modeling the dynamics of novel coronavirus (2019-nCov) with fractional derivative. Alex. Eng. J. (2020)

28. Riaz, M.B., et al.: Couette flows of a viscous fluid with slip effects and non-integer order derivative without singular kernel. Discrete Contin. Dyn. Syst., Ser. S 12(3), 645 (2019) 
29. Baleanu, D., Ghanbari, B., Asad, J.H., Jajarmi, A., Pirouz, H.M.: Planar system-masses in an equilateral triangle: numerical study within fractional calculus. Comput. Model. Eng. Sci. 124(3), 953-968 (2020)

30. Jajarmi, A., Baleanu, D.: A new iterative method for the numerical solution of high-order non-linear fractional boundary value problems. Front. Phys. 8, 220 (2020)

31. Sajjadi, S.S., Baleanu, D., Jajarmi, A., Pirouz, H.M.: A new adaptive synchronization and hyperchaos control of a biological snap oscillator. Chaos Solitons Fractals 138, 109919 (2020)

32. Baleanu, D., Jajarmi, A., Sajjadi, S.S., Asad, J.H.: The fractional features of a harmonic oscillator with position-dependent mass. Commun. Theor. Phys. 72(5), 055002 (2020)

33. Deniz, S.: On the stability analysis of the time-fractional variable order Klein-Gordon equation and some numerical simulations. Commun. Fac. Sci. Univ. Ank. Sér. A1 Math. Stat. 69(1), 981-992 (2020). https://doi.org/10.31801/cfsuasmas.450209

34. Jajarmi, A., Baleanu, D.: On the fractional optimal control problems with a general derivative operator. Asian J. Contro (2019). https://doi.org/10.1002/asjc.2282

35. Mohammadi, F., Moradi, L., Baleanu, D., Jajarmi, A.: A hybrid functions numerical scheme for fractional optimal control problems: application to nonanalytic dynamic systems. J. Vib. Control 24(21), 5030-5043 (2018)

36. Latha, V.P., et al.: A fractional-order model for Ebola virus infection with delayed immune response on heterogeneous complex networks. J. Comput. Appl. Math. 339, 134-146 (2018)

37. Hartley, T.T., Lorenzo, C.F., Killory Qammer, H.: Chaos in a fractional order Chua's system. IEEE Trans. Circuits Syst. I, Fundam. Theory Appl. 42(8), 485-490 (1995)

38. Wei, Q., Wang, X.: Chaos controlling of permanent magnet synchronous motor base on dither signal. J. Vib. Control 19(16), 2541-2550 (2013)

39. Matlob, M.A., Jamali, Y.: The concepts and applications of fractional order differential calculus in modeling of viscoelastic systems: a primer. Crit. Rev. Biomed. Eng. 47(4), 249-276 (2019)

40. Algahtani, O.J.J.: Comparing the Atangana-Baleanu and Caputo-Fabrizio derivative with fractional order: Allen Cahn model. Chaos Solitons Fractals 89, 552-559 (2016)

41. Hu, G., et al.: Controlling spiral waves in a model of two-dimensional arrays of Chua's circuits. Phys. Rev. Lett. 80(9), 1884 (1998)

42. Leonov, G.A., Kuznetsov, N.V.: Analytical-numerical methods for hidden attractors' localization: the 16th Hilbert problem, Aizerman and Kalman conjectures, and Chua circuits. In: Numerical Methods for Differential Equations, Optimization, and Technological Problems, pp. 41-64. Springer, Dordrecht (2013)

43. Deniz, S., Bildik, N.: Optimal perturbation iteration method for Bratu-type problems. J. King Saud Univ., Sci. 30(1), 91-99 (2018)

44. Bildik, N., Deniz, S.: Comparative study between optimal homotopy asymptotic method and perturbation-iteration technique for different types of nonlinear equations. Iran. J. Sci. Technol. Trans. A, Sci. 42(2), 647-654 (2018)

45. Deniz, S.: Optimal perturbation iteration method for solving nonlinear heat transfer equations. J. Heat Transf. 139(7), $074503(2017)$

46. Deniz, S., Bildik, N.: Applications of optimal perturbation iteration method for solving nonlinear differential equations. AIP Conf. Proc. 1798, 020046 (2017)

47. Deniz, S., Bildik, N.: A new analytical technique for solving Lane-Emden type equations arising in astrophysics. Bull. Belg. Math. Soc. Simon Stevin 24(4), 305-320 (2017)

48. Bildik, N., Deniz, S.: New analytic approximate solutions to the generalized regularized long wave equations. Bull. Korean Math. Soc. 55(3), 749-762 (2018)

49. Deniz, S., Konuralp, A., De la Sen, M.: Optimal perturbation iteration method for solving fractional model of damped Burgers' equation. Symmetry 12(6), 958 (2020)

50. Bildik, N., Deniz, S.: A practical method for analytical evaluation of approximate solutions of Fisher's equations. ITM Web Conf. 13, Article ID 01001 (2017)

51. Bildik, N., Deniz, S.: Solving the Burgers' and regularized long wave equations using the new perturbation iteration technique. Numer. Methods Partial Differ. Equ. 34(5), 1489-1501 (2018)

52. Bildik, N., Deniz, S.: New approximate solutions to the nonlinear Klein-Gordon equations using perturbation iteration techniques. Discrete Contin. Dyn. Syst., Ser. S 13(03), 503-518 (2020)

53. Bildik, N., Deniz, S.: A new efficient method for solving delay differential equations and a comparison with other methods. Eur. Phys. J. Plus 132(1), 51 (2017)

54. Deniz, S.: Optimal perturbation iteration method for solving nonlinear Volterra-Fredholm integral equations. Math. Methods Appl. Sci. (2020). https://doi.org/10.1002/mma.6312

55. Deniz, S.: Semi-analytical investigation of modified Boussinesq-Burger equations. J. BAUN Inst. Sci. Technol 22(1), 327-333 (2020)

56. Bildik, N., Deniz, S.: Optimal iterative perturbation technique for solving Jeffery-Hamel flow with high magnetic field and nanoparticle. J. Appl. Anal. Comput. 10(6), 2476-2490 (2020). https://doi.org/10.11948/20190378

57. Deniz, S.: Modification of coupled Drinfel'd-Sokolov-Wilson equation and approximate solutions by optimal perturbation iteration method. AKU J. Sci. Eng. 20(1), 35-40 (2020) 